\title{
Die Entwickelung der Schweissdrüsen in der behaarten Haut des Hundeembryos.
}

\author{
Von \\ Susumu Setooka \\ Aus dem Anatomischen Institut der Keio Universität, Tokyo. \\ (Direktor: Prof. Dr. T. Taniguchi)
}

\section{Einführung.}

Über Untersuchungen der Haut und ihrer Nebenorgane bestehen viele Beschreibungen, so $z$. b. von $\mathrm{Gurlt}$ (1835), Le ydig (1859). H a r m s(1868), Chod ok ow ski(1871), Diem(1907), W i m pf heimer (1907), Tra utmann (1931), Fie bi ger (1931), C l a us he n (1933), $\mathrm{Ba}$ - K i e n-Tsing (1934) deren Spezialität besonders die Haut und die anderen Organe des Hundes ist.

Diese Berichte zeigen Untersuchungen der Schweissdrüse unter einer grossen Anzahl von erwachsenen Hunderassen aber besprachen niemals die Schweissdrüse beim Hundeembryo.

Ich habe die Anzahl der Schweissdrüsen per $1 \mathrm{qcm}$, sowohl deren Form während der Entwickelungsperiode der behaarten Haut studiert.

\section{Material und Methode.}

Das Material welches hier benützt wurde bestand aus 3 HundeEmbryonen einer Bastardart. Diese waren hinsichtlich des Körpergewichtes sowie der Körperlänge untereinander ziemlich gleich.
A. Körpergewicht
$100 \mathrm{~g}$.
Scheitelsteisslänge
$11.5 \mathrm{~cm}$.
B. Körpergewicht
$190 \mathrm{~g}$.
Scheitelsteisslänge
$14 \mathrm{~cm}$.
C. Körpergewicht
$240 \mathrm{~g}$.
Scheitelsteisslänge
$16 \mathrm{~cm}$.

Die Embryonen wurden von der Mutter entfernt gleich nach dem diese getötet, wurde, dann in $10 \%$ Formalin fixiert und in Paraffin eingebettet. Sagittalschnitte von $10 \mu$ Dicke wurden hergerichtet und mit Haematoxylin (Hansen) -Eosin gefärbt. 
Von folgenden 21 Körperteilen wurden Hautstücke ausgeschnitten : Scheitel, Stirn, Hals, Brust, Bauch, Beugeseite des Oberarmes, Streck seite des Oberarms, Beugeseite des Vorderarms, Streckseite des Vorderarms, mediale Seite des Oberschenkels, laterale Seite des Oberschenkels, vordere Seite des Unterschenkels, hintere Seite des Unterschenkels, Rücken, Lenden, Nasenflügel, Oberlippe, Unterlippe Steiss, Achselgrube und Ohrmuschel.

Diese 21 Teile sind beim erwachsenen Hund im allgemeinen stets behaart.

\section{Eigene Befunde.}

Die Zahl der Haaranlage und die der Drüsenanlage in einem Gesichtsfeld gehen im grossen und ganzen mit einander parallel.

Es gibt aber hin und wieder Ausnahmen, wo je nach dem Körperteil das Zahlenverhältnis nicht immer parallel aussieht.

Unter Distanz: versteht man die Entfernung zwischen der Hautoberfläche und der Ausmündungsstelle der Drüsenanlage.

Unter Winkel : versteht man den Winkelraum Raumgehalt welcher zwischen der Haaranlage und der Epidermis besteht.

Scheitel: Die Dichtigkeit der Drüsen und Haaranlage ist mittelmässig. Je nachdem Fortschritt der Entwickelung wird die zuvorerwähnte Distanz kürzer und der Winkel grösser. Die Entwickelung der Haaranlage ist von mittlerer Art.

Die Drüsenanlage zeigt beim “A” Embryo ein nach der stumpfen Winkelseite hin vorspringendes kugeliges Bild.

Selten befindet sich ein hŭgelartiges oder stäbchenartiges Bild, welches in das Korium hineinwächst.

Im B-Tiere zeigt die Schweissdrüsenanlage eine stäbchenartige Form. Beim C-Tiere erweitert sich die Spitze der Schweissdrüsenanlage und weist eine Trommelstockartige Form auf. Die Schweissdrüsenanlage senkt sich sodann nach unten der Haaranlage entlang. Die Spitze der Anlage ist auffallend stark von Haematoxylin gefärbt.

Man sieht die Talgdrüsenanlage kugelförmig oder in einer hängenden Form unter der Ausmündungsstelle der Schweissdrüsenanlage beim "B" und “C" Hunde.

Stirn: Die Dichtigkeit der Schweissdrüsenanlage per $1 \mathrm{~cm}^{2}$ nimmt zu, ihre Distanz wird kürzer, und der Winkel grösser. Aber keiner Schweissdrüsenanlage hängt die Epidermis direkt an.

Die Entwickelung der Haaranlage ist etwas bemerkenswerter als 
die der Schweissdrüsen-Anlage.

Beim A- Hund ist der Winkel zwischen der Schweissdrüsenanlage und der der Epidermis sehr klein und fast vertikal geneigt. Aber je nachdem Entwicklungsgrad vom B- und C- Hund vergrössert sich der Winkel.

Beim A-Hund zeigt die Schweissdrüsenanlage eine kugel-oder stäbchenartige Form.

Der B- Hund lässt in seiner Schweissdrüsenanlage viele stockartige oder stäbchenartige Formen erkennen.

Bei dem C. Hund sieht man in der Schweissdrüsenanlage stäbchenartige oder hakenartige Formen welche mit dünnem Stiele in den Haarbalg ausmünden.

Man begegnet vielen Talgdrüsen beim B- und C- Hund. Halsteil :

Die Veränderungen des Dichtigkeitswinkels und der Distanz in diesem Körperteil ähneln der der Stirn.

Der Grad der Entwickelung bei der Haaranlage scheint normal $z u$ sein. Beim A. Hund sieht man stäbchenartige oder schweiss kugelartige Formen. Bei der kugelartigen Schweissdrüsenanlage, findet man keine Talgdrüsen gerade unter der Schweissdrüsenanlage begeg. net man Talgdrüsen unter der Schweissdrüsenanlage.

Beim B- und C-Hund zeigt die Schweissdrüsenanlage eine schlag. stockartige oder trommelstockartige Form, deren Ausmündungsstelle sehr nahe zur Epidermis steht.

Brustteil :

Die Dichtigkeit ist etwas begrenzt und die Distanz ist schon beim A.Hund kurz. Nach keiner Veränderung des Fortschrittes in der Entwickelung sieht man eine Veränderung der Distanz. Auch im Winkel scheint keine Abänderung erkennbar zu sein.

Der Entwickelungsgrad der Haaranlage ist im allgemeinen normal und die der Schweissdrüsenanlage ist im wesentlichen gleich wie die im Halsteil. Man trifft die Talgdrüsenanlage kugelförmig oder in einer hängenden Form gerade unter der Ausmündungsstelle der Schweissdrüsenanlage beim B- und C- Hunde an. Zuweilen scheint es als ob die Haaranlage ringsum mit einer Talgdrüsenanlage umgeben ist.

Bauchteil :

Die Dichtigkeit ist etwas begrenzt, je nach dem Fortschritt des Entwickelungsgrades wird die Distanz kürzer sowie der Winkel kleiner. Andere Befunde sind dem Halsteil ähnlich. 
Beuge-und Streckseite des Oberarmes :

Der Entwickelungsgrad der Haaranlage ist zeitig später als an anderen Körperteilen. Die Veränderung des Winkels zeigt den gleichen Grad wie die der Stirn. Bei der Streckseite des Oberarmes ist die Dichtigkeit mittelmässig aber bei der Beugeseite macht sie ca die Hälfte der üblichen Haaranlage aus, trotz der reichlichen Haaranlage. Die Distanz wird mit dem Verlauf der Entwickelung in der Beugeseite kürzer. In der Streckseite sind keine nennenswerte Veränderungen bemerkbar. Man kann im grossen und ganzen hügelartige oder kugelartige und eine kleine Menge von stäbchenartigen Schweissdrüsen im A- Hund sehen.

Im B- und C- Hund zeigt die Schweissdrüse eine Schlagstockartige oder trommelstockartige Form, ist nach unten geneigt und scheinbar entlang der Haaranlage kriechend.

Beuge- und Streckseite des Vorderarmes :

Die Dichtigkeit ist etwas grösser. Die Veränderungen in der Distanz und dem Winkel ähneln der am Scheitel. Die Entwickelung der Haaranlage scheint normal zu sein.

Beim A- Hunde, zeigt die Schweissdrüsenanlage eine kugel- oder hügelartige Form, selten die stäbchenartige Form.

Beim B- Hunde, sieht man im allgemeinen stäbchen- oder schlagstockartige Schweissdrüsenanlage und beim C. Hund begegnet man eine stäbchenartige ober schlagstockartige Schweissdrüsenanlage.

Man kann keine Talgdrüsenanlage im A- Hunde finden, jedoch bei dem B- und C- Hund findet man hügel ober kugelartige Talgdrüsen. Innere und äussere Seite des Oberschenkels :

Die Dichtigkeit erscheint mittelmässig und der Winkel verändert sich mit dem Fortschritt des Entwickelungsstadiums aber die Distanz ist schon im A- Hund kurz und keine nennenswerte Veränderung lässt sich in dem Verlauf der Entwickelung erkennen. Die Entwickelung der Haaranlage ist normal. Beim A- Hund zeigt sich die Schweissdrüsenanlage im grossen und ganzen stäbchenartig ober kugelartig.

Beim B- und C- Hund, sieht man die schlagstockartige und trommelstockartige Schweissdrüse.

Vorder- und Hinterteil des Unterschenkels :

Die Entwickelung der Haaranlage verläuft etwas spät. Die Schweissdrüsenanlage zeigt im allgemeinen hügel- oder kugelartige Form beim A- Hund. Die Distanz wird etwas kürzer mit dem Fortschritt des Entwickelungsstadiums. Die übrigen Befunde sind denen 
des Oberschenkels ähnlich.

Rücken :

Die Dichtigkeit der Schweissdrüsenanlage ist gross und die Veränderungen des Winkels und der Distanz zeigen einen normalen Zustand. Der Entwickelungsgrad der Haaranlage verläuft etwas später. Beim AHnnd sieht man in der Schweissdrüsenanlage die stäbchen- ober schlagstockartige Form, und zuweilen begegnet man die, wie ein Bogen gekrümmte, Schweissdrüsenanlage. Der B- und C- Hund lässt eine schlagstock oder trommelstockartige Schweissdrüsenanlage erkennen. Lendenteil :

Man s:eht eine mehrfach gekrümmte stäbchenartige Schweissdrüsenanlage. Die anderweitigen Befunde sind denen des Rückenteils gleich.

Nasenflügelteil :

Die Dichtigkeit ist hochgradig kondensiert. Die Distanz wird immer kürzer mit dem Verlauf des Entwickelungsstadiums.

Die Winkel $z$ wischen der Schweissdrüsenanlage und der Hautoberflache nähert sich seiner Grundrichtung an und vergrössert sich nicht mit dem Fortschritt des Entwickelungsstadiums. Die übrigen Befunde sind normal.

Beim C- Hund senkt sich die Schweissdrüsenanlage tief nach dem Korium hinab, darunter sind auch einige deren Spitze sich hakenförmig biegt.

Ober- und Unterlippe :

Bei der Oberlippe ist die Dichtigkeit etwas gering und bei der Unterlippe mittelmässig. Vom B- bis C- Hund wird die Distanz mit dem Fortschritt des Entwickelungsstadiums kürzer.

Der Winkel wird grösser vom B- zum C- Hund. Die im A- Hund gesehene stäbchen- und hügelartige Schweissdrüsenanlage wird beim B- Hund in der Länge und Breite grösser und zeigt schlagstockartige oder trommelstockartige Form. Bei der Unterlippe zeigt sie eine unregelmässige Form, einige von ihnen weisen eine geigenartige Form auf. Beim C- Hund, senkt die Schweissdrüsenanlage sich noch tiefer und biegend zum Korium hinab. Der Entwickelungsgrad der Talgdrüse ist gut. Die Talgdrüse tritt kugelartig auf beiden Seiten der Haare heraus.

Wurzelteil des Schwanzfortsatzes:

Die Dichtigkeit der Schweissdrüsenanlage ist gross. Die Veränderung des Grades des Winkels ist dem des Scheitels ähnlich.

Die Veränderungen der Distanz zwischen der Schweissdrüsenanlage 
sind erheblich. Beim C- Hund, scheint es als ob die Schweissdrüsenanlage sich direkt an der Hautoberfläche öffnet. Der Entwickelungsgrad der Haaranlage ist schnell, Beim A- Hund zeigt sich die Schweissdrüsenanlage stäbchenartig oder schlagstockartig. Bei dem BHund senkt sie sich tief biegend an dem Korium entlang der Haaranlage. Der C- Hund zeigt die Schweissdrüsenanlage mit einem schmalen Lumen an ihrem Ende. Der Halsteil der Endvergrösserung der Schweissdrüsenanlage ist sehr schmal. (Siehe: Fig. 13. 14. 15.)

Achselteil :

Die Dichtigkeit der Schweissdrüsenanlage ist klein der Entwick. lungsgrad der Haaranlage geht schnell vor sich. Man sieht schon beim A- Hund, dass die Talgdrüsenanlage meistens hügelartig beschaffen ist. Beim A- Hund zeigt die Schweissdrüsenanlage meistenteils stäbchenartige oder schlagstockartige Form. Der B- und C. Hund zeigt das Ende der Schweissdrüsenanlage ziemlich vergrössert. Das Lumen kann man noch nicht sehen.

Dichtigkeit der Haaranlage sieht mittelmässig aus. Die Anzahl der Schweissdrüsenanlage ist klein. Der Winkel nähert sich gegen die senkrechte Richtung in jedem Stadium. Die Schweissdrüsenanlage zeigt beim A- Hund die kugelförmige, hügelartige und stäbchenartige Form.

Beim B- Hund sieht man stäbchenartige ober schlagstockartige Schweissdrüsenanlage. Im C. Hund begegnet man meistens einer schlagstockartigen Schweissdrüsenanlage. Der Entwicklungsgrad der Talgdrüsenanlage ist von normaler Natur.

\section{Besprechung.}

Es ist sehr schwer das Stadium der Ersterscheinung der Schweissdrüsenanlage festzustellen. Nämlich im ein und dem selben Körper ist jede Schweissdrüsenanlage anders im Entwicklungsgrad beschaffen. Im frühen Stadium der Entwicklung ist es sehr schwer die Schweissdrüsenanlage von der Talgdrüsen-anlage $z u$ unterscheiden weil die Formen so sehr einander ähneln. B a ckmund (1904) sagte, dass er im Katzenembryo schon im Stadium des Haarzapfen eine Schweissdrüsenanlage fand. D i e m (1907) fand im Stadium des Haarvorkeimes eine Schweìssdrüsenanlage. $\mathrm{Nag}$ a (1939) fand im menschlichen Embryo von 4 Monaten im Stadium des Haarzapfen eine Schweiss. drüsenanlage.

Ich habe auch selbst eine hügelförmige Schweissdrüsenanlage im 
Übergangsstadium vom Haarzapfen zum Bulbuszapfen gefunden. Die erste Erscheinung der Talgdrüsen-anlage scheint sich erşt später als die der Schweissdrüsenanlage zu zeigen, nämlich die Talgdrüsen-anlage kann das erste mal im Stadium des Bulbuszapfens gesehen werden, gerade unter der Schweissdrüsenanlage.

Über die Stelle der Öffnung der Schweissdrüsenanlage sagte G u r 1 t (1835), dass die Schweissdrüse sich gerade über der Talgdrüse in den Haarbalg öffnet und nicht an der freien Oberfläche des Epitheliums, ausser da wo sich kein Haar vorfindet.

Leydig (1859) teilte mit, dass die Schweissdrüsen sich am oberen Ende des Haarbalges öffnen, In 1895 äusserte Marks, dass alle Schweissdrüsen sich an der Oberfläche der Haut öffnen. Wimpfheimer (1907) meldete, dass die Schweissdrüsen vom Haarfollikel ausgehen und einige der Schweissdrüsen öffnen sich unabhängig an der freien Oberfläche des Epitheliums. In 1934 sagte Ban-Kien-Tsing, dass die Öffnungsstellen der Schweissdrüsen sich am Haarbalg befinden. In dieser Studie fand ich, dass fast sämtliche Schweissdrüsen sich an der Stelle öffnen wo der Winkel zwischen Epidermis und Haarbalg liegt, beim A- Hund auf 100 gr Körpergewicht gesehen und $11.5 \mathrm{~cm}$ in Scheitelsteisslänge.

Beim A- Hund habe ich schon Schweissdrïsen an jedem Teil, welchen ich untersuchte gefunden, das waren 21 Teile des Körpers.

Bei 15 der 21 Teile steigert sich die Öffnungstelle mit dem Verlauf des Entwicklungsstadiums. Beim C- Hund bin ich einem solchen Falle begegnet, da öffneten sich einige Schweissdrüsen direkt an der freien Hautoberfläche an der Wurzel des Schwanzes. Ferner bemerkte ich in anderen Teilen von diesem Körper, dass die Schweissdrüsenanlage sich direkt am Übergangsteil zwischen dem Haarbalg und der Epidermis öffnet.

Über die Beziehung zwischen Schweissdrüsenanlage und Haaranlage.

In 1907 berichteten $\mathrm{Diem}$ und $\mathrm{Wimpfheimer}$, dass eine Haaranlage nur eine Schweissdrüsenanlage im Säugetierembryo besitzt. In dieser Studie habe ich ebenfalls bemerkt, dass zu einem Haarbalg nur eine Schweissdrüsenanlage gehört, und niemals zwei zu einer Haaranlage. Die Zahl der Schweissdrüsenanlage in einem Gesichtsfeld ist aber geringer denn die der Haaranlage. Eine derartige Neigung tritt im frühen Entwicklungsstadium schärfer hervor. Der Winkel zwischen der Haar- und Schweissdrüsenanlage wird grösser je nach dem Fortschritt des Entwicklungsgrades von 17 Teilen an, in 21 Teilen eines Körpers. Demnach neigt der Winkel zwischen der Epidermis und 
Schweissdrüsenanlage sich zu vergrössern.

Über die Form der Schweissdrüsenanlage sagt $\mathrm{Harms}$ (1868), dass der Durchmesser den Endteil der Schweissdrüsenanlage des Hundes nicht verändert, wohl aber die Länge der Schweissdrüsenanlage je nach ihrem Entwicklungsgrad:

Chodokowski (1891) meint, dass die Schweissdrüsen anlaga des Hundeembryos überall in der Haut gesehen wird, aber ihre Form Grösse, der Sitz und ihre Anzahl, verändert sich je nach dem Körperteil und dem Behaarungszustand jeder Rasse.

In unserer Untersuchung begegnet man hügel-, kugel- und stäbchenförmige Schweissdrüsenanlage in jedem Teile des Körpers beim A-Embryo, sehr klein. Bei dem C- Embryo sieht die Schweissdrüsenanlage mittelgross aus, manchmal auch regelrecht gross. Man trifft auch schlagstockartige, trommelstockartige, hakenartige und auch unbestimmte Formen der Schweissdrüsenanlage an. Dann verschmälert sich der Hals der Schweissdrüsenanlage und geht tief in das Corium hinunter und schlängelt sich an der Talgdrüse entlang, beim B-Hund findet man zuweilen eine bogenförmig gekrümmte, stäbchenförmige Schweissdrüsenanlage.

Im Endstadium des Entwicklungsstadium, nämlich beim C- Hund, sieht man schmale Lumen am Endteil der höchstgradig entwickelten Schweissdrüsenanlage. Der Entwicklungsgrad der Schweissdrüsenanlage scheint mit der der Haaranlage parallel zu gehen.

Man sieht zuerst die Talgdrüse am Stadium des Bulbuszapfens der Haarânalage, gerade unter dem Öffnungspunkt der Schweissdrüsenanlage. Die Talgdrüse zeigt zuerst eine Hügelform projektiert dann kugelförmig nach einer oder beiden Seiten der Haaranlage hin und zeigt am Ende des Entwicklungsstadiums eine lappenartige Form.

\section{Zusammenfassung.}

Wie bereits erwähnt habe ich hauptsächlich die apokrine Schweissdrüse von drei Bastardhundeembryonen untersucht jedes Embryo befand sich in einem verschiedenen Entwicklungsstadium. Ich habe die apokrine Schweissdrüse in jedem der 21 Körperteile gefunden. Die Anzahl der Schweissdrüsenanlage und die Dichtigkeit der Haaranlage verändèrt sich je nach dem Körperteil. Ich habe den nach oben geschobenen Öffnungspunkt die Formveränderung der Schweissdrüsenanlage, Vergrösserung des Winkels zwischen der Haar- und Schweissdrüsenanlage je nach dem Fortschritt des Entwicklungs-stadiums 


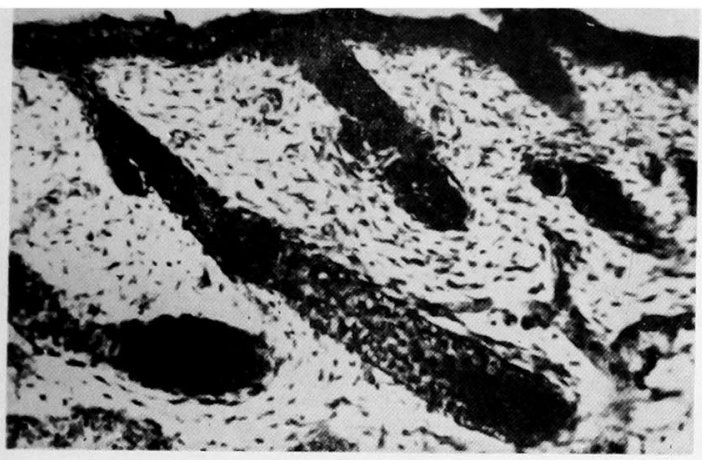

Fig. 1. Streckseite des Oberarms „, A " Embryo

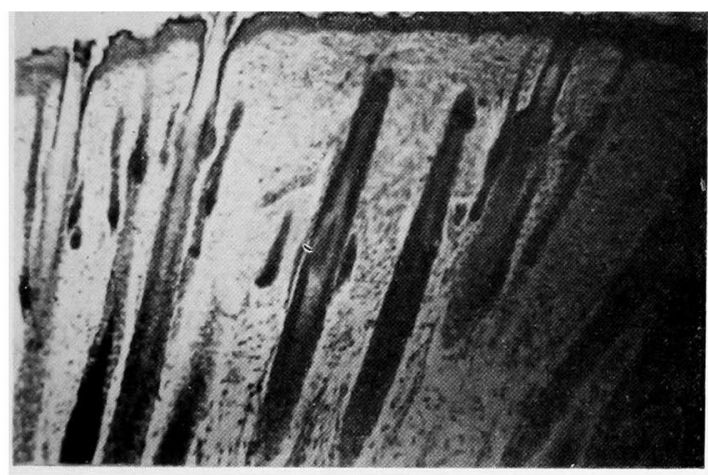

Fig. 2. Streckseite des Oberarms "B" Embryo

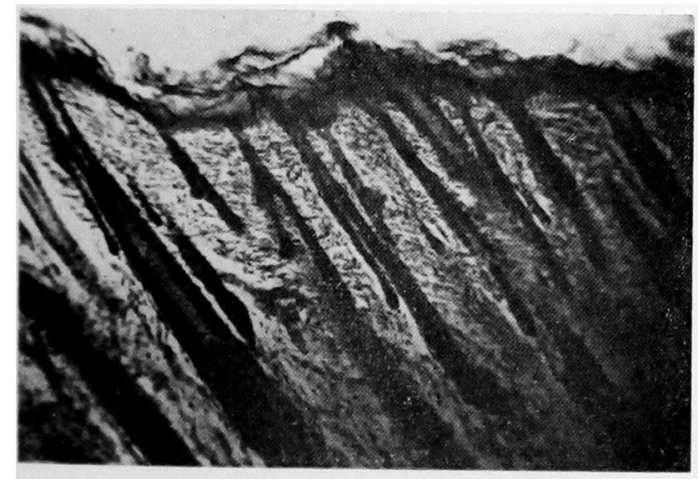

Fig. 3. Streckseite des Oberarms "C" Embryo

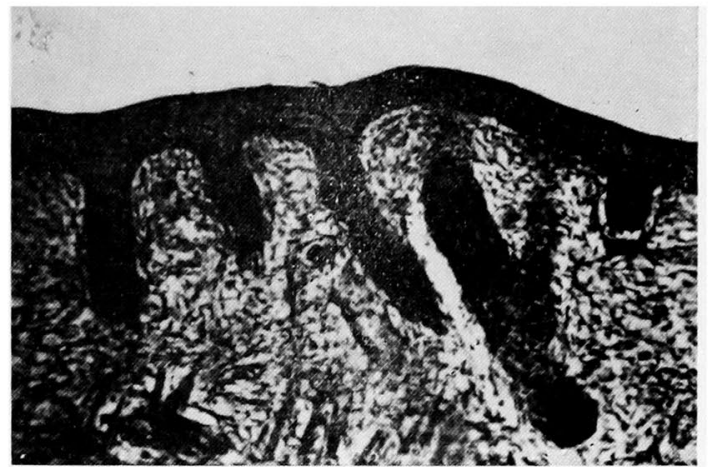

Fig. 4. Beugeseite des Vorderar.ns „A " Embryo

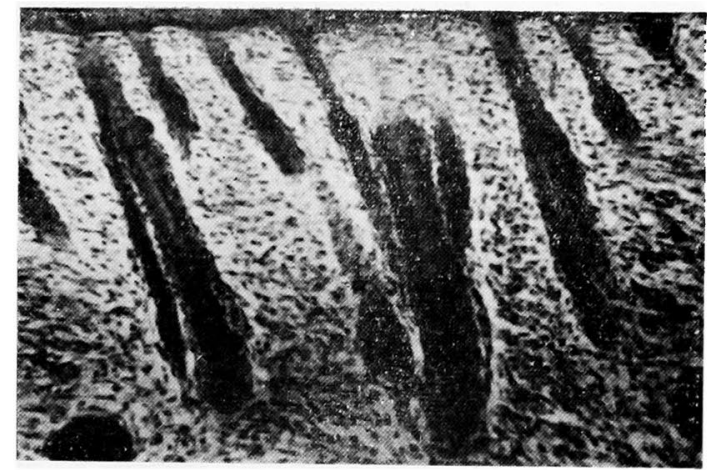

Fig. 5. Beugeseite des Vorderarms „B" Embryo

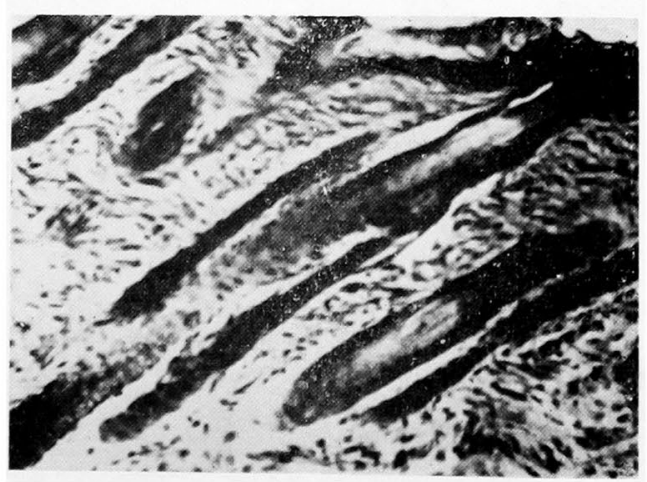

Fig.6. Beugeseite des Vorderarms „C" Embryo 


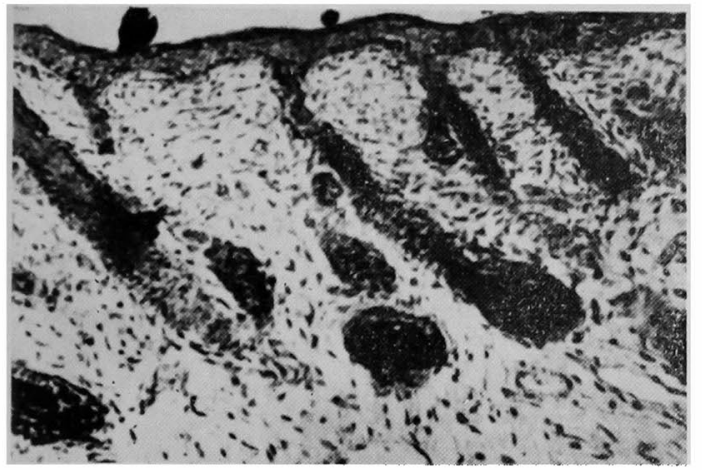

Fig. 7. Mediale Seite des Oberschenkels „A" Embryo

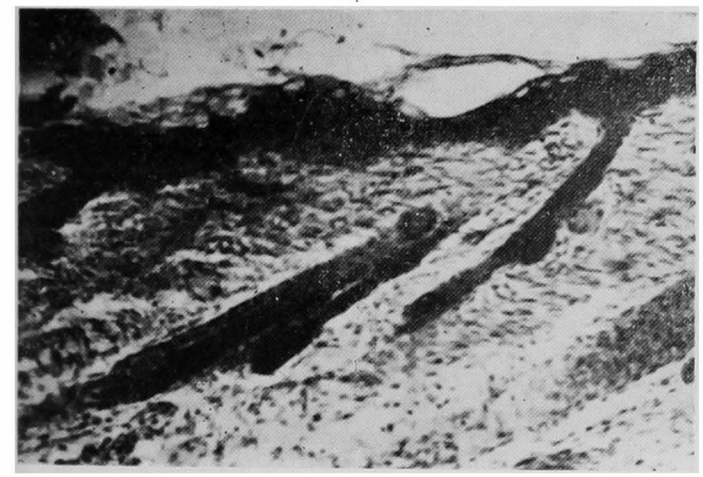

Fig. 8. Mediale Seite des Oberschenkels „B" Embryo

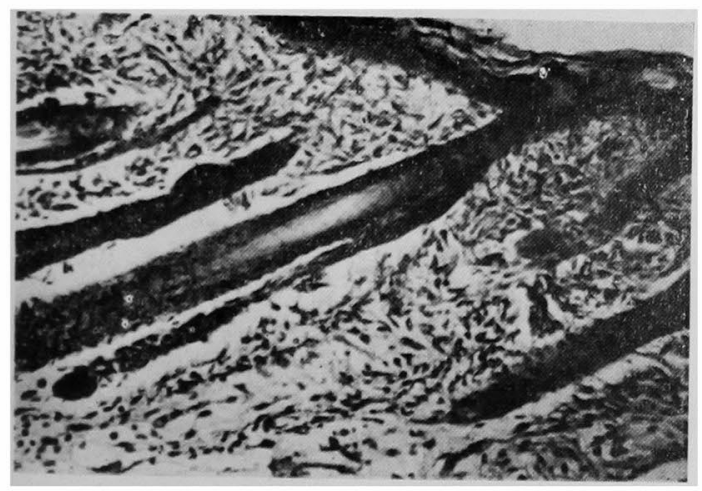

Fig. 9. Mediale Seite des Oberschenkels ,C" Embryo

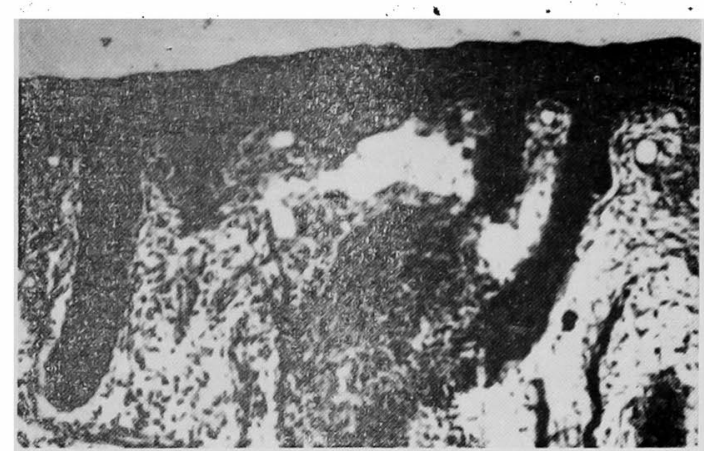

Fig. 10. Unterlippe „A" Embryo
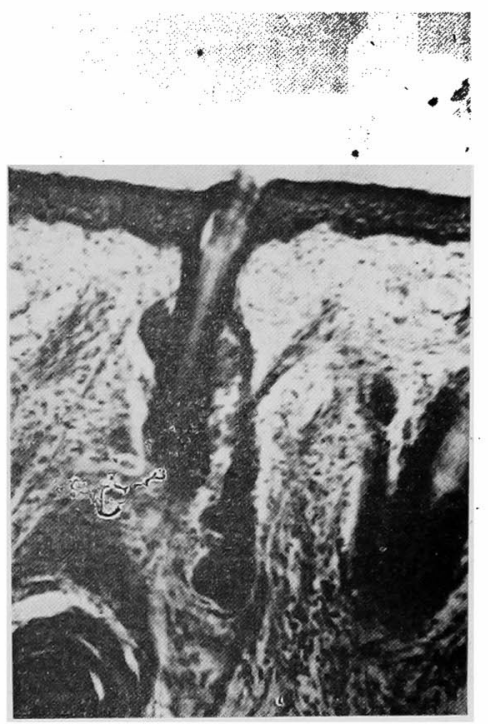

Fig.11. Unterlippe „B” Embryo

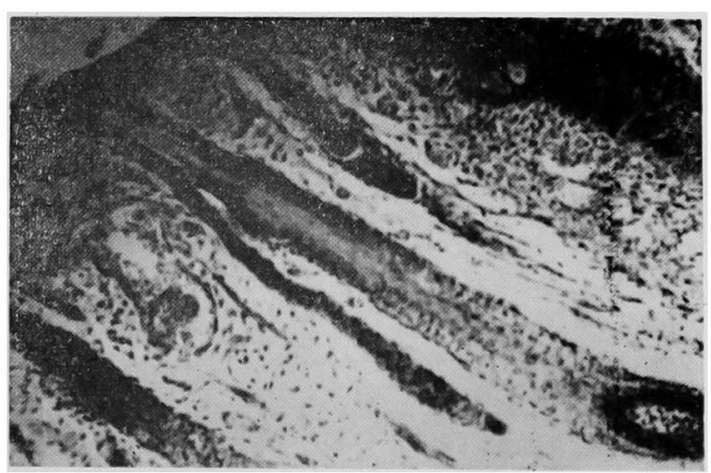

Fig. 12. Unterlippe „C" Embryo 


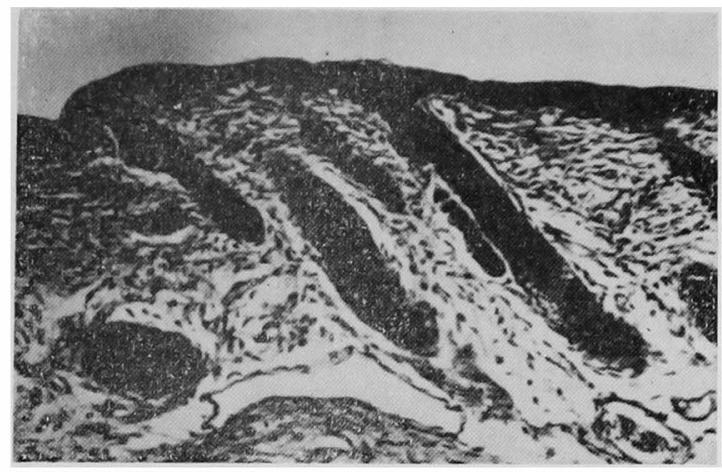

Fig. 13. Steiss „A " Embryo

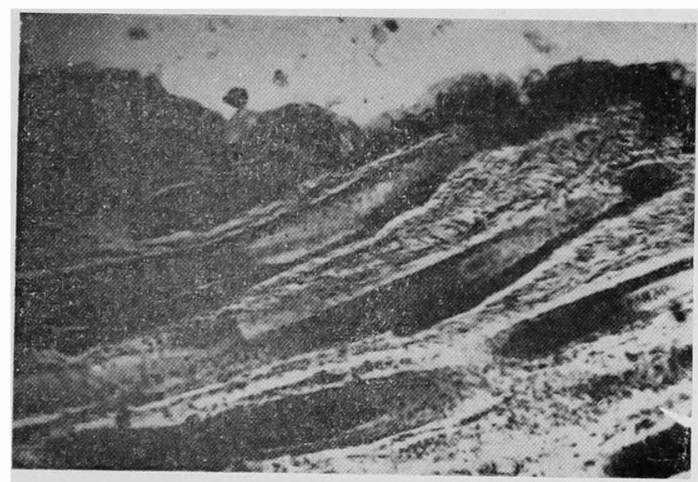

Fig. 14. Steiss „B" Embryo

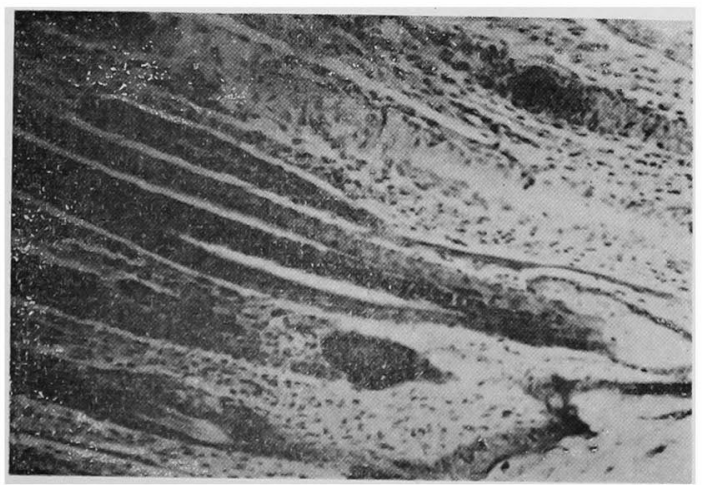

Fig. 15. Steiss "C" Embryo 
gesehen.

Es ist mir ein aufrichtiges Bedürfnis Herrn Prof. Dr. T. Taniguchi für seine freundliche Leitung und sorgsame Kritik meinen besten Dank auszusprechen.

\section{Literatur.}

1. Backmund. K. Entwicklung der Haare und Schweissdrüsen der Katze. Anatomische Hefte. Bd. 26, 1904.

2. B a n-Ki en-Tsing. Über die Schweissdrüse des Hundes. Kaibogaku-Zasshi. Vol. 7, 1934.

3. Cla uschen, A, Mikroskopische Untersuchungeu über die Epidermalgebilde am Rumpfe des Hundes mit besonderer Berücksichtigung der Schweissdrüsen. Anat. Anz. Bd. 77, 1933.

4. Di e m. F. Schweissdrüsen an der behaarten Haut der Säugetiere. Anatomische Hefte. Bd. 34, I907.

5. It o. T. Histologie und Zytologie der Schweissdrüse. Igaku no Schimpo. Vol. 6, 1949. (Japanisch)

6. Wimpfheimer.. C. Zur Entwicklung der Schweissdrüsen der behaarten Haut. Anatomische Hefte. Bd. 34, 1907. 